EPiC Series in Engineering
Volume 3, 2018, Pages 1388-1396
HIC 2018. 13th International
Conference on Hydroinformatics

\title{
Fault-Tolerant Model Predictive Control Applied to Integrated Urban Drainage and Sanitation Systems for Environmental Protection
}

\author{
Jordi Meseguer $^{1 * \dagger}$, Bernat Joseph-Duran ${ }^{1}$, Gabriela Cembrano ${ }^{1,2}$ and \\ Vicenç Puig 2,3 \\ ${ }^{1}$ Cetaqua, Water Technology Center, Carr. Esplugues 75,Cornellà de Llobregat, Spain \\ jmeseguer@cetaqua.com, bjoseph@cetaqua.com \\ ${ }^{2}$ Institut de Robòtica i Informàtica Industrial(CSIC-UPC), Barcelona, Spain \\ cembrano@iri.upc.edu \\ ${ }^{3}$ CS2AC-UPC, Rambla Sant Nebridi 10, Terrassa 08222, Catalunya \\ vicenc.puig@upc. edu \\ Abstract
}

This paper presents a FTC framework for a Real-Time MPC-based Controller applied to Integrated Urban Drainage and Sanitation Systems (UDSSs) which was proposed in the LIFE EFFIDRAIN project. This project deals with the pollution of surface waters due to CSOs and overflows from UDSSs during wet weather. The main purpose of the proposed FTC framework is to preserve as much as possible, the performance of the MPC-based Controller in terms of operation objectives when anomalies affecting the integrated ICT elements (sensors and actuators) occurs. The performance of the FTC controller has been tested using a realistic case of study.

\section{Introduction}

Combined Urban Drainage Networks (UDNs) collect and convey wastewater and storm water together. This mixed water is sent to Waste Water Treatment Plants (WWTPs) where it is treated before being released to the receiving environment. During heavy-rain events, the UDN and WWTP capacities can be easily overloaded, causing untreated water discharges to receiving water bodies, known as combined sewer overflows (CSOs).

In order to prevent CSOs, modern UDNs are equipped with detention elements capable of storing the incoming volume during peak rain instants to later release this volume at lower flow rates, suitable for WWTP treatment. Real-time control (RTC) of these storage tanks can further improve the network capacity to avoid or minimize the impact of CSOs [1].

Urban Drainage and Sanitation Systems (UDSSs) which include UDN and WWTP are highly interconnected systems distributed along large geographical areas making centralised control (RTC) and its performance rely on proper and healthy Information and Communication Technologies (ICT)

* Masterminded EasyChair and created the first stable version of this document

${ }^{\dagger}$ Created the first draft of this document 
Fault Tolerant Model Predictive Control Applied to Integrated Urban Drainage... J. Meseguer et al.

as sensors (i.e. rain gauges, level meters), actuators (pumping station, detention gates) or telecommunication networks. On the other hand, the surrounding conditions found inside UDSSs can potentially lead to sensor or actuator malfunctions, among others [2]. These features imply that RTC applied to UDSSs is sensitive to failure or malfunction of its components and therefore, Fault-Tolerant Control (FTC) strategies must be developed for safe and efficient operation. In this context, a faulttolerant (FT) controller is one which can fulfil the control objectives in spite of the presence of malfunctions, possibly at the expense of some performance loss. A FT framework makes "intelligent" use of the redundancies included in the system (physical) and in the information (analytical) about the system in order to increase the system availability when certain components are affected by faults [3].

This paper presents a FTC framework proposed in the LIFE EFFIDRAIN project [4]. This project deals with the pollution of surface waters due to CSOs and overflows from UDSSs during wet weather. In order to tackle this environmental problem, LIFE EFFIDRAIN develops a RTC methodology [5][6] for the UDSSs based on Model Predictive Control (MPC) following a pollution-based approach: [7]. The main purpose of the proposed FTC framework is to preserve as much as possible, the performance of the MPC-based Controller in terms of operation objectives (environment protection, avoiding flooding and optimizing the use of the WWTP) when anomalies affecting the integrated ICT elements (sensors and actuators) occurs.

This paper is organized as follows: Section 2 presents the proposed FTC framework recalling MPCbased Controller formulation and covering both Fault Diagnosis and Controller Re-design functionalities of this framework. Then, the performance of the proposed FTC framework is assessed using a realistic case of study in Section 3. Finally, main conclusions are discussed in Section 4.

\section{MPC-based Fault-Tolerant Framework}

\subsection{Introduction}

In the LIFE EFFIDRAIN project, the proposed active FTC framework takes benefit from the formalism needed by the MPC-based controller [5][6] applied to the integrated UDSSs for the protection of the environment. Therefore, no new infrastructure is needed to enable and deploy this framework. In that sense, regarding their components [3]:

- Fault Diagnosis is implemented using a model-based approach considering the UDSS simplified model integrated used as constraints in the MPC formalism. This approach allows both Fault Detection based on residual generation and evaluation [8], and Fault Isolation based on an extension of the Fault Signature Matrix concept [9].

- Controller Re-design is implemented using the inherent mechanisms available in the MPC procedure [10] which allow update on-line the MPC problem according to the availability of actuators or sensors diagnosed by the Fault Diagnosis module (On-line Active FTC approach).

\subsection{Real-Time Control: MPC formulation}

Model-predictive control (MPC) is a technique based on solving on-line finite-horizon optimal control problems formulated using the system mathematical model as constraints $\boldsymbol{C}$ and last system measurements as model updates [11]. In general, control objectives $\boldsymbol{O}$ are formulated in terms of an objective function $\boldsymbol{J}$ to be minimized over a time ahead finite- horizon $H$ to compute the optimal control law $\vartheta$. In the MPC methodology, the optimal control problems are solved numerically on-line allowing for the inclusion of additional information to the problem such as constraints on the system variables (i.e., actuator bounds) or disturbance forecasts.

The general Optimal control problem $(O C P)$ formulation which are solved during the operation of the MPC controller at time-step $t$ will be named $O C P(t)$, and is of the form given by Eq. (1). To

\footnotetext{
${ }^{\ddagger}$ Pollution-based RTC has the objective to minimize the total amount of pollutants sent to the receiving water bodies by preferably storing polluted water and spilling more diluted water [7].
} 
distinguish the different problems and their corresponding solutions the time instant in which these problems are formulated and solved is added to each variable. For example $\hat{\boldsymbol{x}}(k \mid t)$ is the value of variable $\hat{\boldsymbol{x}}$ at time-step $k$ as computed (predicted) at time step $t \leq k$ solving the problem $O C P(t)$ at time instant $t$.

$$
\begin{aligned}
& \min _{\boldsymbol{X}(t), U(t)} \boldsymbol{J}(\boldsymbol{X}(t), \boldsymbol{U}(t), \boldsymbol{W}(t)) \\
& \text { s.t. } \\
& \hat{\boldsymbol{x}}(t)=\boldsymbol{x}_{0} \\
& \hat{\boldsymbol{x}}(k+1 \mid t)=\boldsymbol{g}_{1}(\hat{\boldsymbol{x}}(k \mid t), \boldsymbol{u}(k \mid t), \boldsymbol{w}(k \mid t)), \quad k=t, \ldots, t+H-1 \\
& \boldsymbol{g}_{2}(\hat{\boldsymbol{x}}(k \mid t), \boldsymbol{u}(k \mid t), \boldsymbol{w}(k \mid t)) \leq 0, \quad k=t, \ldots, t+H \\
& \boldsymbol{x}_{\min } \leq \hat{\boldsymbol{x}}(k \mid t) \leq \boldsymbol{x}_{\max }, \quad k=t, \ldots, t+H \\
& \boldsymbol{u}_{\min } \leq \boldsymbol{u}(k \mid t) \leq \boldsymbol{u}_{\max }, \quad k=t, \ldots, t+H
\end{aligned}
$$

where $H$ is the time ahead optimization horizon; $\boldsymbol{g}_{1}$ and $\boldsymbol{g}_{2}$ determine the system model; $\boldsymbol{x}_{0}$ is the initial values of the model states at time instant $t$ obtained from the available system measurements; $\boldsymbol{X}(t)=(\hat{\boldsymbol{x}}(t \mid t), \hat{\boldsymbol{x}}(t+1 \mid t), \ldots, \hat{\boldsymbol{x}}(t+H \mid t))$ is the sequence of the system states computed at time instant $t ; \boldsymbol{U}(t)=(\boldsymbol{u}(t \mid t), \boldsymbol{u}(t+1 \mid t), \ldots, \boldsymbol{u}(t+H \mid t))$ is the sequence of the control actions computed at time instant $t ; \boldsymbol{W}(t)=(\boldsymbol{w}(t \mid t), \boldsymbol{w}(t+1 \mid t), \ldots, \boldsymbol{w}(t+H \mid t))$ is the sequence of the disturbances as forecasted at time-step $t$.

This framework is especially suitable to add fault-tolerant capabilities to the controller, since the MPC-based $O C P$ (Eq. (1)) can be updated from one time-step to the next with the information provided by the Fault Diagnosis module (i.e. faulty system component and fault impact). Thereby, tolerance against faults can be embedded relatively easily [10]:

- Modifying bounds on the actuators, $\hat{\boldsymbol{u}}_{\text {min }} \leq \boldsymbol{u}(k \mid t) \leq \hat{\boldsymbol{u}}_{\text {max }}$ (including setting an actuator to a fixed value with $\left.\hat{\boldsymbol{u}}_{\min }=\hat{\boldsymbol{u}}_{\max }\right)$.

- Modifying system model dynamic equations, $\boldsymbol{x}(k+1 \mid t)=\hat{\boldsymbol{g}}_{1}(\hat{\boldsymbol{x}}(k \mid t), \boldsymbol{u}(k \mid t), \boldsymbol{w}(k \mid t))$

- Modifying system model static equations, $\hat{\boldsymbol{g}}_{2}(\hat{\boldsymbol{x}}(k \mid t), \boldsymbol{u}(k \mid t), \boldsymbol{w}(k \mid t)) \leq 0$

- Relaxing nominal control objectives included in the objective function, $\hat{\boldsymbol{J}}(\hat{\boldsymbol{x}}, \boldsymbol{u}, \boldsymbol{w})$, in order to reflect limitation under faulty conditions.

The RT MPC-based controller proposed in LIFE EFFIDRAIN follows the formalism given by Eq. (1). In [6], the process of obtaining UDSS control oriented conceptual hydraulic and water quality models (mainly, flow, level and TSS) is described. These simplified models are suitable to be integrated in the MPC formalism (Eq. (1)) as constraints $\boldsymbol{C}$. Regarding the objective function $\boldsymbol{J}$ representing the control objectives $\boldsymbol{O}$, it is represented by a weighted aggregation of various goals taking into account both operational and environmental purposes:

Quantity Objectives

- $J_{\text {CSO }}$ : Minimizing the CSO discharges from the sewer network to the environment;

- $J_{\text {WWTP: }}$ Maximizing the usage of the WWTP;

- $J_{\text {safe: }}$ : Minimizing flooding and overflows in detection tanks;

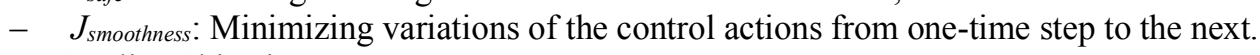
Quality Objectives 
Fault Tolerant Model Predictive Control Applied to Integrated Urban Drainage... J. Meseguer et al.

- $J_{\text {mass: }}$ : Minimizing the pollutant load to the environment (mass of suspended solids in CSO released to the environment).

Considering the above objectives, the objective function $\boldsymbol{J}$ is written as a weighted sum as follows:

$$
J=a_{c s o} J_{c s o}+a_{w w t p} J_{w w t p}+a_{\text {safe }} J_{\text {safe }}+a_{\text {smoothness }} J_{\text {smoothness }}+a_{\text {mass }} J_{\text {mass }}
$$

The weights $a_{C S O}, a_{W W T P}, a_{\text {safe }}, a_{\text {smoothness }}, a_{\text {mass }}$ are chosen taking into account the prioritization of the partial objectives and the numerical range of variation of each of them.

\subsection{Fault Diagnosis}

\subsubsection{Fault Detection}

The proposed fault detection approach is based on an extension of the model-based methodology [9] described in [12] considering interval models with parametric uncertainty [13].

In general, those sensors considered in the proposed FTC approach are those ones needed to initialize the simplified model states $\boldsymbol{x}_{\boldsymbol{0}}$ and the Optimal Control Problem $(O C P(t)$; (Eq. (1)) at every time instant $t$ and those ones measuring actuator outputs and provided by existing local controllers: i.e. level meters as measurements to estimate volumes (i.e. in tanks) or flows (i.e. in sewers) and turbidity meters as measurements of TSS in different UDSS locations; downstream level meters measuring outflows of pumps, detention and redirection gates.

Considering the $O C P$ formulation (Eq. (1)) and the approach proposed in [12], the nominal system output estimations at time instant $t, \hat{\boldsymbol{y}}^{o}(t)$, could be computed when solving the $O C P(t-1)$ at time instant $t-1$ using the nominal simplified model of the UDSS adding the system output model equation:

$$
\hat{\boldsymbol{y}}^{o}(t)=\boldsymbol{h}^{o}\left(\hat{\boldsymbol{x}}^{o}(t \mid t-1), \boldsymbol{u}(t \mid t-1), \boldsymbol{w}(t)\right)
$$

where index ' $o$ ' stands for nominal values. Using the nominal system prediction given by Eq. (3), nominal residuals at time instant $t$ can be computed as follows [9]:

$$
\boldsymbol{r}^{o}(t)=\boldsymbol{y}(t)-\hat{\boldsymbol{y}}^{o}(t)
$$

where $\boldsymbol{y}(t)$ is a vector with the measurements of the system output a time instant $t$. Additionally, considering control actions obtained by solving $O C P(t-1), \boldsymbol{U}(t-1)$ (Eq. (1)), and parametric uncertainty $\boldsymbol{\theta}$ associated with the simplified models, upper and lower bounds for $\hat{\boldsymbol{y}}^{\boldsymbol{o}}(t)$ can be computed as follows:

$$
\hat{\hat{\boldsymbol{y}}}(t)=\min _{\boldsymbol{\theta}}(\hat{\boldsymbol{y}}(t, \boldsymbol{\theta})) \quad \text { and } \quad \overline{\hat{\boldsymbol{y}}}(t)=\max _{\boldsymbol{\theta}}(\hat{\boldsymbol{y}}(t, \boldsymbol{\theta}))
$$

Using Eq. (5), the uncertainty can be propagated to the residual building the interval:

$$
[\underline{\boldsymbol{r}}(t), \overline{\boldsymbol{r}}(t)]
$$

where $\underline{\boldsymbol{r}}(t)=\underline{\hat{\boldsymbol{y}}}(t)-\hat{\boldsymbol{y}}^{o}(t)$ and $\overline{\boldsymbol{r}}(t)=\overline{\hat{\boldsymbol{y}}}(t)-\hat{\boldsymbol{y}}^{o}(t)$

Then, taking into account the definition of the nominal residual Eq. (4) and its associated adaptive threshold Eq. (6), no fault will be indicated while the following fault detection condition is satisfied

$$
\boldsymbol{r}^{o}(t) \in[\underline{\boldsymbol{r}}(t), \overline{\boldsymbol{r}}(t)]
$$

Note the equivalence of this condition and the one expressed in terms of system output measurements and their associated estimation ${ }^{\S}$.

\subsubsection{Fault Isolation}

As initial approach, the considered fault isolation methods are based on an extension of the theoretical fault signature matrix concept, $\boldsymbol{F S M}$, [9]. The fault detection approach described in Section

$$
\S \boldsymbol{y}(t) \in[\underline{\hat{\boldsymbol{y}}}(t), \overline{\hat{\boldsymbol{y}}}(t)]
$$


Fault Tolerant Model Predictive Control Applied to Integrated Urban Drainage... J. Meseguer et al.

2.3.1 allows to define a residual (Eq. (4)) per each existing sensor. Then, taking into account the simplified models of the UDSSs integrated in the MPC formalism (Eq. (1)), the explicit equations of the existing residuals could be derived and consequently, matrix $F S M$ could be obtained understood as the Cartesian product of the sets of faults $\boldsymbol{f}=\left\{f_{j}: j=1,2, \ldots, n_{f}\right\}$ and fault signals $\phi=\left\{\phi_{i}: i=1,2, \ldots . n_{\phi}\right.$ \} derived from the residual test given by Eq. (7) [9]. In this first approach, binary $\boldsymbol{F S M}$ has been considered meaning $\phi_{i} \in\{0,1\}$ and if the element $\boldsymbol{F S M}_{i j}$ of this matrix is equal to ' 1 ', it means the fault $f_{j}$ causes the occurrence of the fault signal $\phi_{i}$; otherwise, the element $\boldsymbol{F S M}_{i j}$ is equal to ' 0 '. The real values of every component $\phi_{i}$ of the observed fault signature $\phi$ can be computed at time instant $t$ as follows:

$$
\varnothing_{i}(t)=\left\{\begin{array}{l}
0 \rightarrow r_{i}^{o}(\mathrm{t}) \in\left[\underline{r}_{i}(t), \bar{r}_{i}(t)\right] \\
1 \rightarrow r_{i}^{o}(t) \notin\left[\underline{r}_{i}(\mathrm{t}), \bar{r}_{i}(t)\right]
\end{array}\right.
$$

\subsection{MPC-based Fault Tolerant Strategies}

\subsubsection{Introduction}

This section presents the FTC strategies to be activated by the Controller Re-design module once a fault is diagnosed. According to the proposed FTC framework (Section 2.1) and the MPC formalism (Eq. (1)), once a sensor fault is diagnosed at time instant $t$, its measurement needs to be estimated online so that the $O C P(t)$ problem can be initialized properly (Section 2.2). Regarding a fault affecting an actuator, once it is diagnosed at time instant $t$, the $O C P(t)$ problem needs also to be updated on-line to inform the total or partial unavailability of the given actuator using the proper mechanism of the MPC formalism: mainly, the constraints associated with $O C P(t)$ (Section 2.2).

\subsubsection{Sensor Fault Strategies}

The proposed approaches take benefit from the MPC formalism and the UDSS simplified models integrated as constraints (Eq. (1)). As a basic approach, for a fault affecting the measurement $y_{i}(t)$ for $t \geq t$, the faulty measurement is substituted by the estimation obtained when solving the control problem $O C P(t-1)$ and applying Eq. (3):

$$
y_{i}(t) \approx \hat{y}_{i}^{o}(t) \text { for } t \geq t_{f}
$$

Nonetheless, in order to initialize the control problem $O C P(t)$ (Section 2.2), the following relation could be applied directly:

$$
x_{i}(t) \approx \hat{x}_{i}^{o}(t \mid t-1) \text { for } t \geq t_{f}
$$

As a drawback, the estimation error given by the assumption Eq. (9) may be propagated over the time. This negative impact may have a major influence on systems with fast dynamics. Another applied approach to estimate measurements of faulty sensors could be based on solving a Moving-Horizon Estimation (MHE) problem [14] using a slight modification of the $O C P(t)$ solved at every MPC iteration. The MHE is a finite horizon optimization-based observer which provides approximations of all the system variables by minimizing the difference between past measurements and the corresponding values obtained by the system model.

\subsubsection{Actuator Fault Strategies}

As mentioned in Section 2.1, the FTC strategies applied when an actuator fault is diagnosed are based on reconfiguring the MPC-based controller reflecting on-line the total or partial unavailability of the faulty actuator. Then, the controller tries to find existing redundancy in the other available actuators to compensate the impact of the fault while trying to keep performance as high as possible. This can be done through the on-line modification at every iteration of the $O C P$ constraints determined by the UDSS 
simplified model to take into account actuator faults impact. Depending on the type of actuator and the type of fault, the $O C P$ constraint are modified in a different way.

For the case of a degraded performance or breakdown in a pumping group at time instant $t \geq t$, a constraint is added to the $O C P$ s to take into account that the maximum available flow capacity of the group has dropped from the nominal maximum flow $Q_{p}^{\max }$ to a lower value $\hat{Q}_{p}^{\max }<Q_{p}^{\max }$.

$$
0 \leq Q_{p}(k) \leq \hat{Q}_{p}^{\max }, \quad k=t, \ldots, t+H, \quad t \geq t_{f}
$$

where $Q_{p}\left[\mathrm{~m}^{3} / \mathrm{s}\right]$ is the flow pumped by the pumping group. When the group is no longer available, constraint given by Eq. (11) could be re-written as $Q_{p}(t)=0, t \geq t$ :

Following a similar reasoning process, new constraints could be identified to represent degraded performance of other actuators as detention or re-direction gates.

\section{Case of Study}

\subsection{Description}

Document The Test Network (Figure 1) is composed by a pumping group designed to pump all the inflow to a WWTP in dry weather (Pumping Group 2). In wet weather, two additional pumping groups (Group 1 and Group 3) can pump additional inflow to CSO in order to keep the infrastructure safe. The whole control station consists of three pumping groups, three tanks and several big sewers with considerable inline storage capacity. Additionally, several level meter sensors are also available (green spots in Figure 1).

The assessment of the considered fault scenario has been carried out considering three cases: the MPC case, where the system is controlled using a MPC controller and NO failure occurs at all; the MPC + Fault case where the system is controlled with the same MPC controller and a fault occurs; and the FTMPC case where FT strategy described in Section 2.4.3 is applied updating the $O C P$ constraints related to the faulty group once the fault is detected.

These three cases have been further combined with three different rain events of variable intensity: low-intensity (Scenario 1), high-intensity (Scenario 2) and medium-intensity with a long duration (Scenario 3).

\subsection{MPC model and control objectives}

Figure 2 shows the simplified model of the control station used as constraints in the MPC formalism (MPC model). In this model, the tank volume not only takes into account the storage capacity of the three tanks in the station but also the storage capacity of the upstream sewers. This allows the computed control strategies to keep the CSO pumps off until the stored volume is close to the safety level which also considers the retention capacity of the sewers.

Regarding the objective function (2), the considered weights give high priority to avoid flooding and overflows in the detention tank while avoiding CSOs and maximizing the WWTP usage have a second priority: $a_{C S O}=10, a_{W W T P}=10, a_{\text {safe }}=1000, a_{\text {smoothness }}=1, a_{\text {mass }}=0$. In this test, the quality term of the objective function, $J_{\text {mass }}$, has not been considered.

\subsection{Fault scenario analysis}

The fault scenario studied in the Test Network consist in a failure on an actuator: CSO Pumping Group 1 (Figure 1 and 2). The considered failure is a complete breakdown of the element, which is assumed to stop working. The failure starts 2 hours after the rain event start and lasts until the end of the simulation, 12 hours later. If no corrective action is taken the control algorithm will continue sending positive flow set-points to the pumping group that cannot be accomplished. Therefore the tank is not emptying at the flow rates predicted by the controller, which can eventually lead to flooding. 
Fault Tolerant Model Predictive Control Applied to Integrated Urban Drainage... J. Meseguer et al.

Considering the Scenario 2 associated with a rain event of high intensity, in Figure 3, upper plot, the evolution over the time of the measured and predicted (nominal ${ }^{* *}$ and interval ${ }^{\dagger \dagger}$ model ) flow of the faulty pump (CSO Pumping 1) has been drawn while in the lower plot, the evolution of the nominal residual and its associated can be seen (7) showing clearly the detection time instant of the fault at time step 24, 2 hours after the rain event started. Once, the fault is detected, the FTC strategy described in Section 2.4.3 could be applied indicating the non-availability of the CSO Pumping 1 Group in the next $O C P(t)$ problems. As a result, MPC-based Controller will make a more intensive use of CSO Pumping 2 Group.

In Table 1, the values of the CSO, WWTP treated water and flooding volumes are computed considering the three cases and the three rain scenarios. In the MPC+Fault case, it can be seen that the fault lead to big flooding volumes. However, in the FTC controller (FTMPC case), MPC-based Controller knows the non-availability of CSO Pumping 1 Group suggesting to use more intensively CSO Pumping 2 Group recovering the initial performance (MPC case).

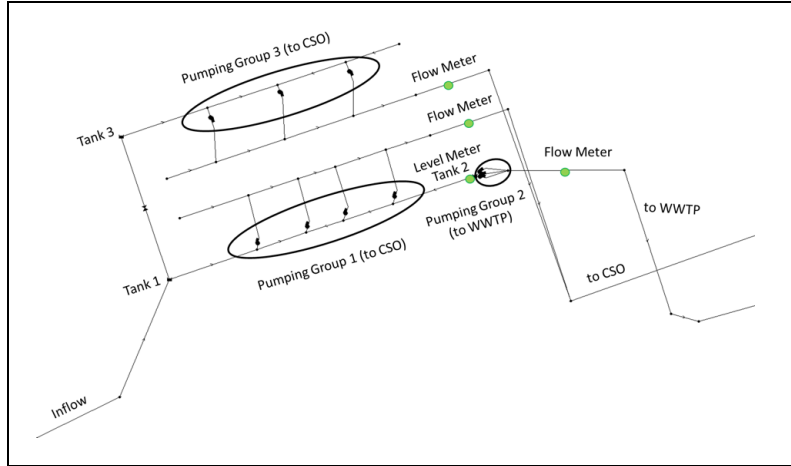

Figure 1: Pumping station in the test network with two pumping groups pumping to $\mathrm{CSO}$ and a third one pumping to WWTP

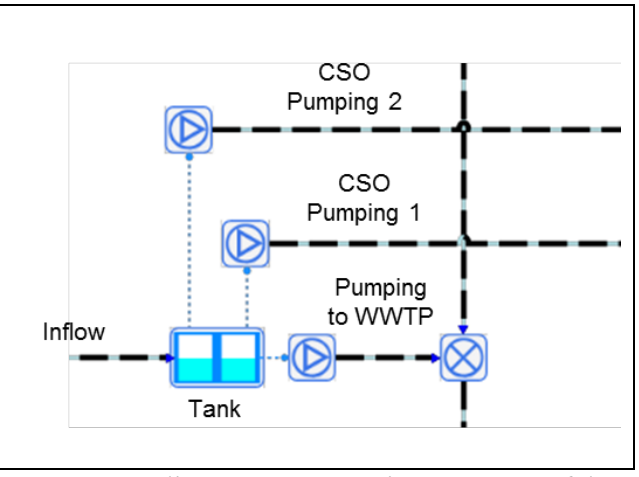

Figure 2: Inline storage capacity upstream of the Control Station in the Test Network

\section{Conclusions}

This paper presents an on-line active FTC framework to preserve as much as possible, the performance of the MPC-based Controller applied to the integrated UDSSs in terms of operation objectives (environment protection, avoiding flooding and optimize the use of the WWTP) when anomalies affecting the integrated ICT elements (sensors and actuators) occurs. The proposed FTC framework takes benefit from the formalism needed by the MPC-based controller. On-line Fault Diagnosis is implemented following a model-based approach using the UDSS simplified model integrated in the MPC formalism while Controller Re-design is based on inherent FT mechanisms available in the MPC procedure. The proposed FTC framework has been tested in a realistic case of study showing an appealing performance.

\footnotetext{
** The prediction is obtained solving $O C P(t-1)$ and is given by the set-point computed for $t-1: \hat{\boldsymbol{y}}_{u}^{o}(\boldsymbol{t})=u(t-1 \mid t-1)$.
}

${ }^{\dagger \dagger}$ An exploration of the historical values associated with the actuator downstream sensor in relation with the actuator set-points has been carried out to set the model parametric uncertainty. As a result the lower and upper bounds of the nominal residual has been obtained. 
Fault Tolerant Model Predictive Control Applied to Integrated Urban Drainage... J. Meseguer et al.

\section{Acknowledgment}

The authors wish to thank the support received by the European Commission research grant of project LIFE EFFIDRAIN (LIFE14 ENV/ES/000860). The authors also want to thank Bordeaux Metropole and Aigües de Barcelona for the financial and technical support.

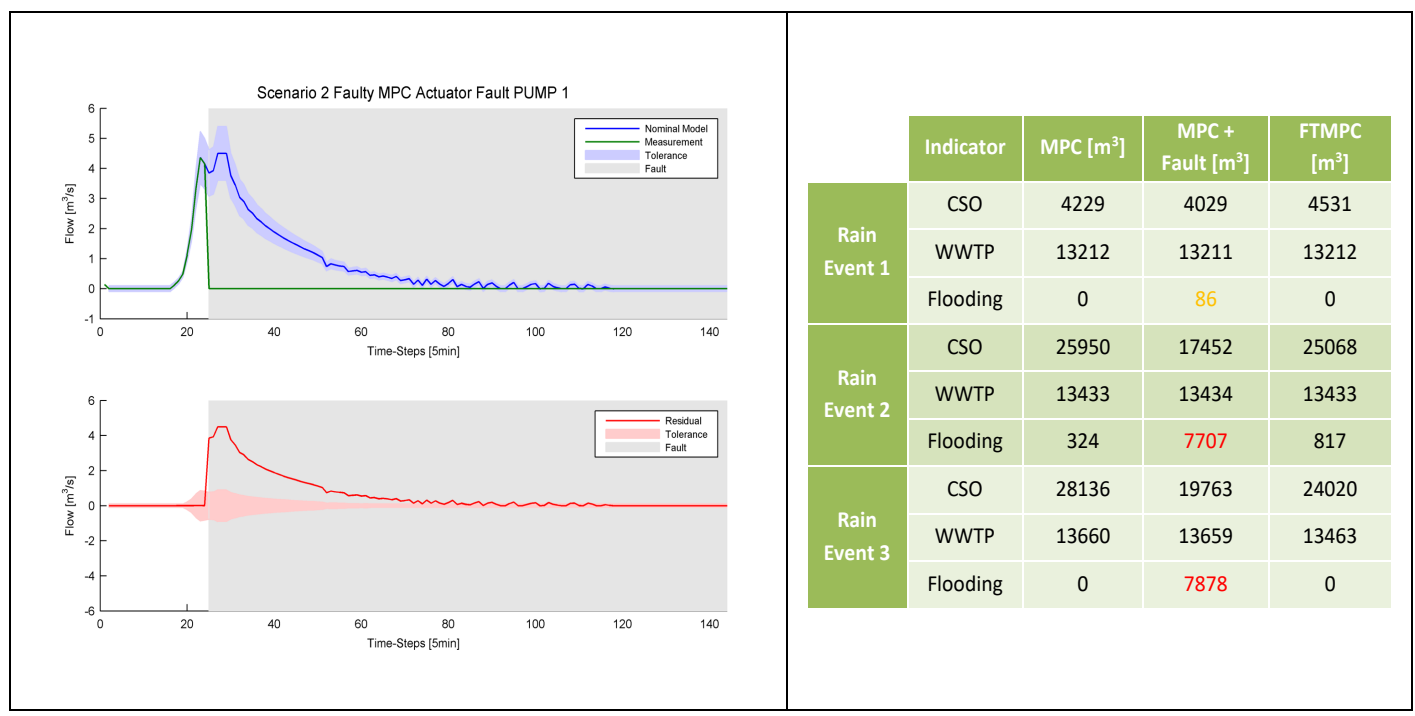

Figure 3: Time evolution of the measured and predicted flow of the faulty pump and the corresponding residual.
Table 1: Performance indicators for the nonfaulty MPC, the faulty MPC scenarios and the FTC Scenarios.

\section{References}

[1] Schütze, M., A. Campisano, H. Colas, W. Schilling, and P. Vanrolleghem (2004), Real time control of urban wastewater systems - where do we stand today?, J. Hydrol., 299(3-4), 335-348

[2] Campisano C., Cabot Ple J., Muschalla D., Pleau M., and Vanrolleghem P.A. (2013) Potential and limitations of modern equipment for real time control of urban wastewater systems. Urban Water J., 10(5), 300-311, doi: 10.1080/1573062X.2013.763996.

[3] Blanke, M., Kinnaert, M., Lunze, J., Staroswiecki, M., and Schröder, J. (2006). Diagnosis and fault-tolerant control (Vol. 691). Springer, Berlin.

[4] Joseph-Duran B., Meseguer J., Cembrano G., Maruéjouls T., Montserrat A., Martínez M., Guasch R., Rouge P. (2016) LIFE EFFIDRAIN: Efficient Integrated Real-time Control in Urban Drainage Systems for Environmental Protection. 14th International CCWI Conference

[5] Puig V., Cembrano G., Romera J., Quevedo J., Aznar B., Ramón G. \& Cabot J. 2009 Predictive optimal control of sewer networks using CORAL tool: application to Riera Blanca Catchment in Barcelona. Water Science \& Technology, 60(4): 869-878

[6] C. Sun, B. Joseph-Duran, T. Maruejouls, G. Cembrano, E. Muñoz, J. Meseguer and V. Puig. Efficient Integrated Model Predictive Control of Urban Drainage Systems using Simplified Conceptual Quality Models. 14th IWA International Conference on Urban Drainage (ICUD). Prage, Czech Republic, 2017.

[7] Vanrolleghem, P. A., Benedetti, L., and Meirlaen, J. (2005). Modelling and real-time control of the integrated urban wastewater system. Environmental Modelling \& Software, 20(4), 427-442, doi: 10.1016/j.envsoft.2004.02.004.

[8] Chow, E. Y. and Willsky, A. S. (1980) "Issues in the development of a general algorithm for reliable failure detection", Proc. of the 19th Conf. on Decission and Control, Albuquerque, NM.

[9] Gertler J.J. (1998) "Fault Detection and Diagnosis in Engineering Systems." Marcel Dekker. 1998 
Fault Tolerant Model Predictive Control Applied to Integrated Urban Drainage... J. Meseguer et al.

[10] Maciejowski,J.(2002). Predictive control with constraints. Great Britain: Prentice Hall.

[11] Camacho; Bordons (2004). Model predictive control. Springer Verlag

[12] Meseguer, J., Puig, V., and Escobet, T. (2010). Fault diagnosis using a timed discrete-event approach based on interval observers: Application to sewer networks. IEEE Transactions on Systems, Man, and CyberneticsPart A: Systems and Humans, 40(5), 900-916.

[13] Puig, V., Stancu, A., Quevedo, J. (2005) "Set versus Trajectory based Approaches to Interval Observation". Conference on Decision and Control \& European Control Conference (CDC-ECC'05). Sevilla. Spain.

[14] Rawlings, J. B. (2014). Moving horizon estimation. Encyclopedia of Systems and Control, 1-7. 